\title{
The effect of familiarity on rotational transformation
}

\author{
HOWARD S. HOCK and KIM ROSS \\ Florida Atlantic University, Boca Raton, Florida $\$ \$ 492$
}

\begin{abstract}
Familiarity, established by preliminary reproduction training with unfamiliar dot patterns, decreased "same" reaction time in a same-different comparison task. The familiarity effect was significantly greater when the pairs of patterns were in different orientations (transformational matches), as opposed to the same orientation (exact matches). This interaction between familiarity and the type-of-match supported the hypothesis that familiarity would facilitate the mental rotation of the patterns. In addition, a significant correlation between the effects of symmetry (symmetrical vs. asymmetrical patterns) and rotation (familiar vs. rotated familiar patterns) was obtained for exact matches, replicating Hock's (1973) findings. This evidence for individual differences in processing was the basis for concluding that familiarity had facilitated mental rotation, and not just attention to the orientationally invariant distinctive features of the patterns.
\end{abstract}

Recent research of Cooper and Shepard (1973), Shepard and Feng (1972), and Shepard and Metzler (1971) has provided evidence that subjects can respond correctly on tasks involving mental transformation of the stimulus information, and that the reaction time for the response increases with the amount of transformation demanded by the task. Shepard and Feng's (1972) task required mental paper folding, while Cooper and Shepard (1973) and Shepard and Metzler (1971) both required that their subjects carry out rotational transformations. The latter two studies constitute the basis for the present research.

Shepard and Metzler (1971) used a same-different comparison task. Their stimuli were drawings of unfamiliar, three-dimensional shapes that were constructed by sequentially attaching solid cubes to form rigid, arm-like figures. They presented these shapes in pairs, varying the angular separation between the two members of the pair. When different, the two members of the pair were mirror images of each other. Since the featural information is very similar for mirror-image stimuli, it was argued that the possibility of "same" matches being based on a comparison of rotationally invariant features was minimized. Shepard and Metzler found that the time required for a "same" response to a pair of identical shapes was linearly related to the angular separation between them. The linear relation was obtained when the rotational transformation was in the frontal plane or in the depth plane of the observer.

Using a somewhat different task, Cooper and Shepard (1973) required their subjects to decice

This research was supported by a Seed grant from Florida Atlantic University. Portions of this study were presented at a meeting of the Psychonomic Society, Boston, 1974. Requests for reprints should be sent to Howard S. Hock, Department of Psychology, Florida Atlantic University, Boca Raton, Florida 33432. whether a stimulus was a normal alphabet letter or a mirror-image version of an alphabet letter. As in Shepard and Metzler's study, the mirror-image discrimination minimized the possibility that the subject's decision was based on the detection of rotationally invariant features. They found that response time increased monotonically, but nonlinearly, with increasing angular departure of the letter from its upright orientation.

Although the two studies described above involved different experimental tasks and used stimuli of differing complexity, a comparison of their results suggested that rotational transformations were performed more rapidly for familiar stimuli (alphabet letters) than for unfamiliar stimuli (cube shapes). The possibility that familiarity facilitates mental rotation was the hypothesis of primary concern in the present study. The hypothesis was tested in a same-different comparison task, with familiar and unfamiliar stimuli matched in complexity.

Of further concern was the mode of processing underlying the perception of the stimuli in the mental rotation task. In a series of experiments, Hock (1973), Hock, Gordon, and Gold (1975), and Hock, Gordon, and Marcus (1974) have obtained consistent differences in subjects' mode of processing visual information. These findings are relevant to the mental rotation task since they involve individual differences in the effect, on "same" reaction time, of presenting pairs of familiar stimuli in an unfamiliar orientation.

Hock (1973), using a same-different comparison task, found a significant positive correlation between the effects of symmetry (symmetrical vs. asymmetrical patterns) and rotation (familiar vs. rotated-familiar patterns) on "same" reaction time. It was concluded that those subjects with large symmetry effects, as well as large rotation effects, had emphasized a structural model of processing, while those subjects with small symmetry effects, as well as small rotation effects, had 
emphasized an analytic mode of processing. In subsequent research. Hock. Gordon, and Marcus (1974) found that subjects with small rotation effects (analytic subjects) detected embedded figures more easily than subjects with large rotation effects (structural subjects). and Hock. Gordon, and Gold (1975) found that subjects with small rotation effects (analytic subjects) based their "same" responses to pairs of phrsically identical stimuli on a comparison of verbal codes, while subjects with large rotation effects (structural subjects) based their same responses on a perceptual comparison.

The evidence for individual differences described above points to the same problem faced by Shepard and Metzler: the possibility that some subjects would base their transformational matches ("same" responses to pairs of identical stimuli in different orientations) on a comparison of orientationally invariant features, instead of mentally rotating one stimulus into the orientation of the second. Shepard and Metzler dealt with this problem by using mirror images to form "different" pairs, and assuming that the mirror images were sufficiently similar to rule out the possibility that subjects were attending to orientationally invariant features.

The present study dealt with this problem more directly by examining individual differences in the processing of exact matches (identical stimuli in the same orientation) and distinguishing between analytic subjects, who customarily exhibit orientational invariance. and structural subjects, whose exact matches of familiar stimuli depend on the spatial orientation of the stimuli. Since they do not attend to spatially invariant stimulus properties. some sort of transformational process would be required for structural subjects to match identical stimuli in different orientations. On this basis. if the transformational matches of structural subjects were faster for familiar than for unfamiliar stimuli. it would support the hypothesis that familiarity facilitates mental rotation. Since the same result for analytic subjects could also mean that familiarity facilitates attention to orientationally invariant features of the stimuli. the hypothesized effect of familiarity on rotational transformation was tested only for the subjects emphasizing structural processes.

\section{METHOD}

\section{Stimuli}

The stimuli used in this experiment were composed of the same 20 unfamiliar dot patterns used by Hock (1973). ${ }^{1}$ Each pattern was composed of eight dots. with each dot located in one of 25 possible positions defined by a 5 by 5 grid. Half the patterns were simmetrical and half were asymmetrical, with all the symmetrical patterns having the same axis of symmetry extending from the upper left to the lower right corners of the $5 \mathrm{by} 5$ grid. Restrictions in the construction of the patterns. as well as procedures used to match the simmetrical and asummetrical patterns with respect to their preexperimental tamiliarity. imilarity. and central dispersion. are described by Hock $\left(14^{-} .3\right)$.

\section{Design}

As in Hock (1973). the 20 dot patterns were divided into two subsets of 10 patterns, with each subset comprising 5 symmetrical and 5 asymmetrical patterns. The subset of 10 patterns assigned to the familiar condition was presented during a preliminary training sessions as well as during the experimental session; the subset of 10 patterns assigned to the untamiliar condition was presented only during the experimental session. Since the assignment of the two subsets to the two familiarity conditions was counterbalanced between two groups of subjects. each stimulus appeared equally often in both the faniliar and unfamiliar conditions. and the familiar and unfaniliar stimuli were perfectly matched in complexity.

During the main experimental session, the dot patterns were presented in pairs. For halt the trials. the paired patterns were the same, though they were sometimes presented in different crientations: for the other half. the paired patterns were different. The patterns paired logether were always drawn from the same familiarity condition as well as the same symmetry condition. For example. a symmetrical pattern assigned to the unfaniliar condition could either be paired with itself, to form a "same" pair, or with one of the other four symmetrical patterns assigned to the unfamiliar condition, to form a "different" pair.

Finally, the stimulus pairs differed with respect to the orientation of the dot patterns. The experimental conditions were as follows: (1) both familiat patterias were presented in the orientation in which thes were learned. F(0.0): (2) both familiar patterns were rotated $y$ ) deg comberclockwise into an untamiliar orientation. $F(x),(x)):(3)$ the familiar pattern on the left was in its familiat orientation and the familiar pattern $t(1$ its right was rotated 90 deg counterchekuise. F(O. $(x)$ ): (t) both unfamiliar patterns were presented in their upright orientation. $U(0.0)$ : and (5) the unfamilat pattern on the left was in its upright orientation and the unfaniliat pattern to its right was rotated $\mathscr{Q}$ ) deg counterclockwise. L:(10.)(2) . ${ }^{2}$

The live conditions descriled above were orthogonal with respect to the stmmetry variable (semmetrical is. asymmetrical) and same w. difterent comparison. The "exact" matches for this experiment, which constituted these "same" stimuli for which the two patterns were aluabs in the same orientation. $F(0,0), F(9), 9(0)$. U(0.0). were iclentical with those used by Hock (1973). The transformational matches for this experinent constituted these "same" stimuli for which the two patterns were in different orientations: $F(0,90)$ and ['(1). (x)). Faamples of exact matches (same orientation) and transtormational matches (different orientation) are presented in Fïgure 1.

\section{Procedure}

The procedure used in this experiment also paralleled Hocks $\left.114^{-} .3\right\}$ study. The first step was to sensitize the subjects to the atypical diagonal axis of symmetry. The subjects were sequentially shown the 10 patterns that would later be presented for familiarization training, and the axis of symmetry was denoted for the 5 smmetrical pattems. These 10 patterns were then seciuentially presented in a series of randomized blocks of 10 , the subjess being requited to report whether or not the exposed pattern was symmetrical. The stimuli were presented with a Carousel projector. and exposute times were tachistoscopically controlled at 2.0 sec for the lirst bleck, and at 1.0, 1.0. 0.3. and 0.3 sec for succeeding blocks of triak.

The subjects were then familiarized with the same 10 patterns during 12 blocks of trials in which they were required to learn to reproduce them with perfect accuracy. Following a s-sec exposure of each pattern. the subjects were required to reproduce the pattern on a blank 5 by 5 grid, guessing if thev were unsure of the location of some wh the dots. Cortectise information was prosided after each incorrect reproduction. Fach of the 12 blocks of trials comprised a different random sequence of 10 trials. one for each pattern. Only thase subjects with 9 nut of 10 correct reproductions on the las hock af trials were retained for the remainder of the experimemt.

On the next dats. the subjects were presented with two more blusk of 10 repreductions. for which they received no corrective 


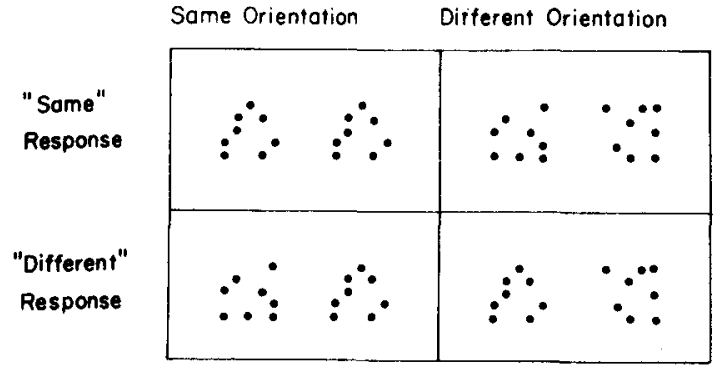

Figure 1. Example of a "same" and "different" pair for patterns presented in the same orientation (exact matches) and patterns presented in different orientations (transformational matches).

feedback. This was followed by the main experimental task, which involved the same-different comparison of pairs of dot patterns. The subjects were told that they would be seeing pairs of patterns they had been reproducing as well as new patterns, and were shown the various orientations in which the patterns would appear. They were told that half the pairs consisted of the same patterns and half of different patterns, and were instructed to press a button, with a finger of their preferred hands, whenever the two patterns in a pair were the same, even when they were presented in different orientations. When the patterns were different. they were not to respond in any way. The subjects were instructed to respond as quickly as possible, within the context of keeping their errors to a mininum.

The stimuli were back-projected onto a translucent screen by a Carousel projector. The duration of each stimulus presentation. which was controlled by a tachistoscopic shutter, was $5 \mathrm{sec}$, unless the subject responded. whereupon the exposure was terminated. The visual angle intercepted by each pattern was $1.2 \mathrm{deg}$, and the overall visual angle was $3.5 \mathrm{deg}$. The subjects received 80 practice trials with pairs of dot patterns that were later repeated as the last 80 trials of the experimental sequence. This was followed by a sequence of 200 experimental trials in which the stimuli from the various experimental conditions were presented in a randomly mixed order.

\section{Subjects}

Tuenty-four unpaid volunteer subjects participated in both days of the experiment. The subjects were undergraduate students at Florida Atlantic University as well as college-age nonstudents.

\section{RESULTS}

Each reproduction obtained during familiarization training was scored on an all-or-none basis; it was either right or wrong. Since a block of reproduction trials consisted of five symmetrical and five asymmetrical patterns, each block had a maximum score of 5 for each kind of pattern. One subject failed to pass the criterion of 9 out of 10 correct on the last block of trials on Day 1. The data for this subject, who was dropped from the experiment, is not included in Figure 2, which summarizes reproduction performance on both days of the experiment.

As can be seen in Figure 2, the initial superiority in reproducing the symmetrical patterns disappeared by the end of Day 1, but reappeared for the two blocks of reproductions at the start of Day 2. The difference between symmetricals and asymmetricals on Day 2's reproductions was significant, $\mathrm{t}(23)=3.84, \mathrm{p}<.001$.

The mean "same" reaction times and percentage errors for the same-different comparison task are presented in Table 1 . With reaction times for the $F(90,90)$ condition excluded, analysis of variance indicated that the effects of familiarity ( $F$ vs. U), $F(1.23)=26.15$, symmetry ( $\mathrm{S}$ vs. $\mathrm{A}), \mathrm{F}(1,23)=$ 71.98 , and type of match (exact vs. transformational), $\mathrm{F}(1,23)=171.46$, were all significant, $\mathrm{p}<.005$. The interaction between familiarity and type of match was significant, $F(1,23)=19.00, \mathrm{p}<.005$, supporting the hypothesis that familiarity would facilitate rotational transformations. In addition, the interaction between symmetry and type of match was significant, $F(1,23)=43.37, \mathrm{p}<.005$, indicating that symmetry served as a useful cue for indicating when a transformational match was required, thereby improving performance on the transformational matches. Finally, the interaction between familiarity and symmetry was insignificant, $F(1,23)<1.0$.

While the above interaction between familiarity and type of match supported the hypothesis for this experiment, it was necessary to rule out the possibility that the significant interaction was due exclusively to subjects who attended to orientationally invariant features of the stimuli. For such subjects, transformational matches could be based on feature comparison, and not mental rotation.

Based on Hock's (1973) findings, individual differences in the mode of processing underlying "same" responses were examined by comptuing both the effect of symmetry, A-S, and the effect of rotation, $F(90,90)-F(0,0)$, for the exact-match reaction times of each subject. ${ }^{3}$ The correlation between the effects of symmetry and rotation was significant, $r=0.41$, $\mathrm{p}<.05$, replicating Hock's $(1973)$ results. ${ }^{4}$ Without the two outlying subjects, indicated by the triangles in the scattergram of Figure 3, the correlation would have increased to $r=0.59$.

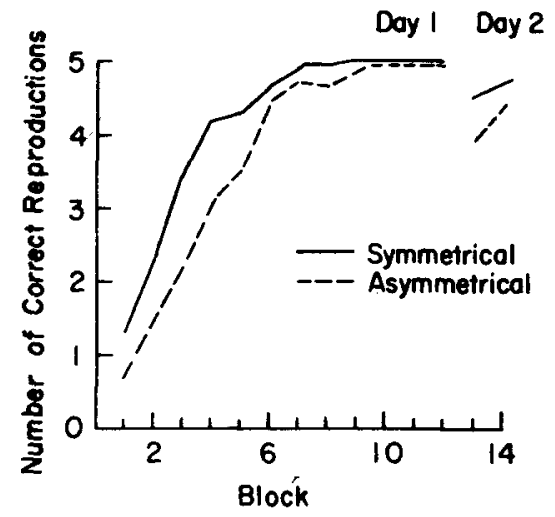

Figure 2. Reproduction data. There were 10 trials in each block, 5 symmetrical and 5 asymmetrical. 
Table 1

Mean "Same" Reaction Time (in Milliseconds) and Percentage Errors for All 24 Subjects

\begin{tabular}{|c|c|c|c|c|c|c|c|c|c|}
\hline & & \multicolumn{4}{|c|}{ Reaction Time } & \multicolumn{4}{|c|}{ Errors } \\
\hline & & \multirow{2}{*}{$\begin{array}{c}\text { Sym } \\
(\mathrm{S})\end{array}$} & \multirow{2}{*}{$\begin{array}{l}\text { Asym } \\
\text { (A) }\end{array}$} & \multirow[b]{2}{*}{$A-S$} & \multirow[b]{2}{*}{$A+S / 2$} & \multicolumn{2}{|c|}{ Omissions } & \multicolumn{2}{|c|}{ Commissions } \\
\hline & & & & & & $\mathrm{S}$ & $\mathbf{A}$ & $\mathrm{S}$ & A \\
\hline \multirow{3}{*}{$\begin{array}{l}\text { Same Orientation } \\
\text { (Exact Match) }\end{array}$} & $F(90,90)$ & 1195 & 1581 & 386 & 1388 & .4 & .4 & 3.3 & 6.7 \\
\hline & $F(0,0)$ & 1154 & 1403 & 249 & 1279 & 0 & 0 & 1.7 & 5.0 \\
\hline & $\mathrm{U}(0,0)$ & 1298 & 1508 & 210 & 1403 & 0 & 0 & .8 & 5.4 \\
\hline Different Orientation & $F(0,90)$ & 1695 & 2403 & 706 & 2050 & 1.3 & 9.6 & 6.3 & 4.2 \\
\hline (Transformational Match) & $\mathrm{U}(0,90)$ & 2054 & 2662 & 608 & 2358 & 2.1 & 9.6 & 1.7 & 4.6 \\
\hline
\end{tabular}

As in Hock's (1973) study, the subjects were divided into two groups on the basis of the size of their symmetry effects for the exact matches. The 12 subjects with the largest symmetry effects (Group 1) were inferred to emphasize a structural mode of processing, while the 12 subjects with the smallest symmetry effects (Group 2) were inferred to emphasize an analytic mode of processing (the median symmetry effect was $285 \mathrm{msec}$. The mean reaction times and percentage errors for the two groups of subjects are presented in Table 2.

As in Hock's (1973) study, familiarity ( $F$ vs. U) significantly affected exact-match reaction time, $F(1,22)=9.87, p<.01$, and tests of simple effects were significant for both the group of structural subjects, $t(22)=4.24, p<.001$, and the group of analytic subjects, $\mathrm{t}(22)=2.10 . \mathrm{p}<.05$. Also replicating Hock (1973), exact-match reaction times for the structural subjects were virtually equivalent for the rotated-familiar, $F(90,90)$ and unfamiliar, $U(0,0)$, stimuli.

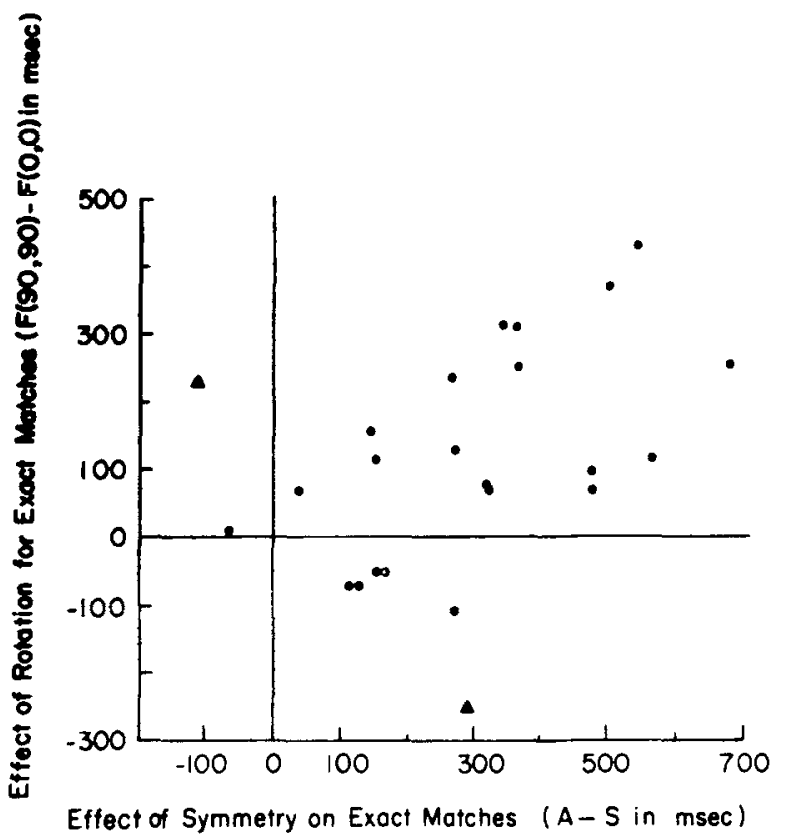

Figure 3. Scattergram for "same" responses. Each point represents the data for one subject. The two outlying subjects are indicated by triangles.
For the group of 12 structural subjects, analysis of variance. with the $F(90,90)$ condition excluded. indicated that the effects of familiarity ( $F$ vs. $U)$, $F(1,11)=15.72$. symmetry (S vs. $A), F(1.11)=$ 76.71, and type of match $(0,0$ vs. 0,90$), F(1,11)=$ 98.77. were all significant. $p<.005$. The interaction between familiarity and type of match was significant, $F(1,11)=17.19, p<.005$, as was the interaction between symmetry and type of match. $F(1,11)=$ 14.01, $p<.005$. The interaction between familiarity and symmetry $u$ as insignificant. $F(1.11)<1.0$, and the higher order interaction between familiarity, symmetry. and type of match was also insignificant. $F(1,13)<1.0$. The results for the structural subjects were the same when the one subject in this group with a negative rotation effect for exact matches (the outlying subject in the lower right quadrant of Figure 3) was excluded from the analysis of variance.

A similar analysis for the group of 12 analytic subjects yielded similar results, the one exception being that the interaction between familiarity and type of match fell short of significance, $F(1.11)=$ 4.75. $\mathrm{p}>.05$. Although the mean rotation effect for exact matches was larger than in Hock's (1973) study, an examination of Figure 3 indicates that half the subjects in the analytic group had either small or negative rotation effects. As discussed above. orientational invariance in the processing of exact matches makes it impossible to determine whether the subjects in the analytic group mentally rotated the patterns or whether they based their transformational matches on a comparison of orientationally invariant features.

Errors of omission were few, with the exception of the transformational matches $(0,90)$ of asymmetrical patterns. For the group of subjects emphasizing structural processes, errors of commission were greater for asymmetrical than for symmetrical patterns, but there was no suggestion that the interaction between familiarity and type of match or the interaction between symmetry and type of match were due to differential speed-accuracy criteria. For the subjects emphasizing analytic processes, however, a high concentration of commission errors was obtained for the transformational matches of symmetrical patterns. This suggested that the signiticant interaction of type of match with 
Table 2

Mean "Same" Reaction Time (in Milliseconds) and Percentage Errors, With Subjects Divided According to Individual Differences in Processing the Exact Matches

\begin{tabular}{|c|c|c|c|c|c|c|c|c|c|}
\hline & & \multicolumn{4}{|c|}{ Reaction Time } & \multicolumn{4}{|c|}{ Errors } \\
\hline & & \multirow{2}{*}{$\begin{array}{c}\text { Sym } \\
(\mathrm{S})\end{array}$} & \multirow{2}{*}{$\begin{array}{c}\text { Asym } \\
\text { (A) }\end{array}$} & \multirow[b]{2}{*}{$A-S$} & \multirow[b]{2}{*}{$\mathrm{A}+\mathrm{S} / 2$} & \multicolumn{2}{|c|}{ Omissions } & \multicolumn{2}{|c|}{ Commission } \\
\hline & & & & & & S & A & $\mathrm{S}$ & $\mathbf{A}$ \\
\hline & & \multicolumn{8}{|c|}{ Group 1 (12 Structural Subjects) } \\
\hline $\begin{array}{l}\text { Same Orientation } \\
\text { (Exact Match) }\end{array}$ & $\begin{array}{l}F(90,90) \\
F(0,0) \\
U(0,0)\end{array}$ & $\begin{array}{l}1306 \\
1208 \\
1432\end{array}$ & $\begin{array}{l}1879 \\
1631 \\
1743\end{array}$ & $\begin{array}{l}573 \\
423 \\
311\end{array}$ & $\begin{array}{l}1593 \\
1420 \\
1588\end{array}$ & $0^{.8}$ & $\begin{array}{l}.8 \\
0\end{array}$ & $\begin{array}{l}2.5 \\
1.7 \\
0\end{array}$ & $\begin{array}{l}5.0 \\
4.2 \\
5.8\end{array}$ \\
\hline $\begin{array}{l}\text { Different Orientation } \\
\text { (Transformational Match) }\end{array}$ & $\begin{array}{l}F(0,90) \\
U(0,90)\end{array}$ & $\begin{array}{l}1717 \\
2173\end{array}$ & $\begin{array}{l}2586 \\
2928\end{array}$ & $\begin{array}{l}869 \\
755\end{array}$ & $\begin{array}{l}2152 \\
2551\end{array}$ & $\begin{array}{r}.8 \\
2.5\end{array}$ & $\begin{array}{r}9.2 \\
10.0\end{array}$ & $\begin{array}{l}2.5 \\
1.7\end{array}$ & $\begin{array}{l}4.2 \\
5.0\end{array}$ \\
\hline & & \multicolumn{8}{|c|}{ Group 2 (12 Analytic Subjects) } \\
\hline $\begin{array}{l}\text { Same Orientation } \\
\text { (Exact Match) }\end{array}$ & $\begin{array}{l}F(90,90) \\
F(0,0) \\
U(0,0)\end{array}$ & $\begin{array}{l}1084 \\
1101 \\
1164\end{array}$ & $\begin{array}{l}1302 \\
1176 \\
1274\end{array}$ & $\begin{array}{r}218 \\
75 \\
110\end{array}$ & $\begin{array}{l}1193 \\
1139 \\
1219\end{array}$ & $\begin{array}{l}0 \\
0 \\
0\end{array}$ & $\begin{array}{l}0 \\
0 \\
0\end{array}$ & $\begin{array}{l}4.2 \\
1.7 \\
1.7\end{array}$ & $\begin{array}{l}8.3 \\
5.8 \\
5.0\end{array}$ \\
\hline $\begin{array}{l}\text { Different Orientation } \\
\text { (Transformational Match) }\end{array}$ & $\begin{array}{l}F(0,90) \\
U(0,90)\end{array}$ & $\begin{array}{l}1677 \\
1935\end{array}$ & $\begin{array}{l}2221 \\
2396\end{array}$ & $\begin{array}{l}544 \\
461\end{array}$ & $\begin{array}{l}1949 \\
2166\end{array}$ & $\begin{array}{l}1.7 \\
1.7\end{array}$ & $\begin{array}{r}10.0 \\
9.2\end{array}$ & $\begin{array}{r}10.0 \\
1.7\end{array}$ & $\begin{array}{l}4.2 \\
4.2\end{array}$ \\
\hline
\end{tabular}

symmetry, and the tendency toward interaction between type of match and familiarity, may have been due, in part. to differential speed-accuracy criteria for the subjects in the analytic group.

\section{DISCUSSION}

Unlike the research of Shepard and collaborators, there was no attempt in this study to establish a linear relation between response time and the amount of required transformation, and to infer from such a relation that subjects were mentally rotating the stimuli. Also unlike Shepard and collaborators, the possibility that subjects were basing their transformational matches on a comparison of orientationally invariant features was not dealt with by using mirror images as "different" pairs and assuming they were sufficiently similar to rule out feature comparison. The approach of this study was to separate subjects who based their "same" responses on a comparison of orientationally invariant features (analytic subjects) from subjects whose "same" responses depend on the orientation of the stimuli (structural subjects). This separation was justified by the signiticant positive correlation between the effects of orientation, $F(90,90)$ $F(0,0)$, and symmetry, A-S, on exact-match reaction time. replicating Hock's (1973) evidence for individual differences in perceptual processing. The orientational dependence of structural subjects, which was also observed by Hock, Gordon, and Gold (1975) and Hock, Gordon, and Marcus (1974), indicates that, unlike analytic subjects, structural subjects are not attentive to orientationally invariant properties of the stimulus. On this basis, it was concluded that structural subjects must carry out some sort of transformational operation, rotation being most likely, in order to correctly respond to transformational matches.
As can be seen in Table 2, familiarity had a large (and significant) effect on transformational-match reaction time, $U(0,90)-F(0,90)$, for the structural subjects. This effect could be attributed to either the influence of familiarity on the processing of the upright pattern in the $F(0,90)$ stimulus or the influence of familiarity on the rotational transformation of the upright pattern into the orientation of the rotated pattern. These alternatives were resolved by the significant interaction between familiarity and type of match for the structural subjects. The familiarity effect for these subjects was significantly larger for transformational than for exact matches, indicating that familiarity had facilitated transformational matches to a greater extent than could be attributed to the effect of familiarity on the processing of the upright pattern in the $F(0,90)$ stimulus. The hypothesis that familiarity would facilitate rotational transformation was therefore supported, but only for subjects emphasizing a structural mode of processing. As discussed previously, the hypothesis could not be evaluated for analytic subjects since these subjects could base their transformational matches on a comparison of orientationally invariant features instead of mentally rotating the patterns.

Although alternative explanations are possible, the results obtained for the subjects emphasizing structural processes are consistent with the view that perceptual learning for structural subjects involves embedding a stimulus in a spatial context by schematically coordinating the information in the stimulus with the subjects' subjective axes of space. On this basis, presenting a familiar stimulus in an unfamiliar orientation would eliminate familiarity effects because the relation between the stimulus information and its spatial context would be disrupted (as in exact matches). When a stimulus is recognized in its upright orientation, however, its coordination 
with a spatial reference would facilitate spatial transformations of the stimulus information (as in transformational matches).

For subjects emphasizing analytic processes, rotating a familiar stimulus into an unfamiliar orientation had a relatively small effect on exact-match reaction time. This invariance. however. did not carry over to the transformational matches. It can be seen in Table 2 that the transformational matches of familiar patterns, $F(0.90)$, were considerably slower than the exact matches of familiar patterns, $F(0,0)$ or $F(90,90)$, for the analytic subjects. If rotational invariance was complete, the analytic subjects would have been equally fast for the exact and transformational matches of familiar patterns.

The results for analytic subjects suggest. therefore. that they are attending to rotationally invariant parts of the patterns, for example, a group of dots forming a right angle in the upper left corner of a pattern. While a right angle is itself rotationally invariant, its location relative to the rest of the pattern is not. This would not affect performance for the analytic subjects if they could detect the right angle in one pattern and tind it in the same location in the second pattern. as would be the case for exact matches of both familiar and rotated-familiar patterns. For transformation matches, however, the analytic subjects may not easily find the right angle in the second pattern since the right angle would be rotated into a different position relative to the first pattern. Whether analytic subjects find the features necessary for transformational matches by a systematic or random search is a question requiring further investigation.

\section{REFERENCES}

Cooper, L. A.. A Shepard. R. N. Chronometric studies of the rotation of mental images. In W. G. Chase (Ed.). Visual infurmarion processing. New York: Academic Press. 1973.

Hock. H. S. The effects of stimulus structure and familiarity on same-difterent comparison. Perception \& Psychophysics. 1973, 14. 41.3-420.

Hock, H. S. Gordon. G. P.. \& Gold. L. Individual differences in the verbal coding of familiar visual stimuli. Memon \& Cognition. 1975, 3. 257.261.

Hock. H. S., Gordon. G. P.. \& Marcus, N. Individual differences in the detection of embedded figures. Perception \& Psychophysics. 1974, 15, 47-52.

MCNemar. Q. Psychological statistics. New York: Wiley, 1962.

Shepard. R. N., \& FENG, C. A chronometric study of mental paper folding. Cognitive Psychology. 1972. 3. 228-243.

Shepard, R. N.. \& Metzier. J. Mental rotation of threedimensional objects. Science. 1971. 171. 701.703.

\section{NOTES}

1. The $20 \mathrm{dot}$ patterns used in this study are presented in Figure 1 of Hock (1973).

2. Upright for the unfamiliar condition corresponded to the orientation of the patterns when they were assigned to the upright familiar condition.

3. The rutation effect was computed by averaging across the symmetrical and as mmetrical patterns. The symmetry effect was computed by averaging over the thre exact-match conditions. $F(0,0), F(90,9))$, and $U(0,0)$.

4. When pertormance kevel. as determined by each subject's exact-match reaction time to the tamiliar. symmetrical patterns. was partialed out (McNemar. 1\%2). the correlation coefficient renlained unchanged.

\{ Received for publication January 15, 1975: revision received April 4. 1975.) 